\title{
Household-level factors associated with relapse following discharge from treatment for moderate acute malnutrition
}

\author{
Heather C. Stobaugh ${ }^{1,2,3 *}$, Beatrice L. Rogers ${ }^{2}$, Patrick Webb ${ }^{2}$, Irwin H. Rosenberg ${ }^{2}$, \\ Chrissie Thakwalakwa ${ }^{4}$, Kenneth M. Maleta ${ }^{4}$, Indi Trehan ${ }^{1,5,6}$ and Mark J. Manary ${ }^{1,4,7}$ \\ ${ }^{1}$ Department of Pediatrics, Washington University in St. Louis, 660 S Euclid Avenue, St. Louis, MO 63110, USA \\ ${ }^{2}$ Friedman School of Nutrition Science and Policy, Tufts University, 150 Harrison Avenue, Boston, MA O2111, USA \\ ${ }^{3}$ RTI International, 3040 E. Cornwallis Road, Research Triangle Park, NC 27709-2194, USA \\ ${ }^{4}$ School of Public Health and Family Medicine, University of Malawi, Private Bag 360, Chichiri, Blantyre 3, Malawi \\ ${ }^{5}$ Department of Pediatrics and Child Health, University of Malawi, Private Bag 360, Chichiri, Blantyre 3, Malawi \\ ${ }^{6}$ Lao Friends Hospital for Children, Luang Prabang 06000, Laos \\ ${ }^{7}$ Children's Nutrition Research Center, Baylor College of Medicine, 1100 Bates Avenue, Houston, TX 77030, USA \\ (Submitted 7 October 2017 - Final revision received 22 January 2018 - Accepted 23 January 2018 - First published online 5 March 2018)
}

\begin{abstract}
Factors associated with relapse among children who are discharged after reaching a threshold denoted 'recovered' from moderate acute malnutrition (MAM) are not well understood. The aim of this study was to identify factors associated with sustained recovery, defined as maintaining a mid-upper-arm circumference $\geq 12.5 \mathrm{~cm}$ for 1 year after release from treatment. On the basis of an observational study design, we analysed data from an in-depth household $(\mathrm{HH})$ survey on a sub-sample of participants within a larger cluster randomised controlled trial (cRCT) that followed up children for 1 year after recovery from MAM. Out of 1497 children participating in the cRCT, a subset of 315 children participated in this sub-study. Accounting for other factors, HH with fitted lids on water storage containers $(P=0 \cdot 004)$ was a significant predictor of sustained recovery. In addition, sustained recovery was better among children whose caregivers were observed to have clean hands $(P=0.053)$ and in HH using an improved sanitation facility $(P=0.083)$. By contrast, socio-economic status and infant and young child feeding practices at the time of discharge and HH food security throughout the follow-up period were not significant. Given these results, we hypothesise that improved water, sanitation and hygiene conditions in tandem with management of MAM through supplemental feeding programmes have the possibility to decrease relapse following recovery from MAM. Furthermore, the absence of associations between relapse and nearly all HH-level factors indicates that the causal factors of relapse may be related mostly to the child's individual, underlying health and nutrition status.
\end{abstract}

Keywords: Moderate acute malnutrition: Supplemental feeding programmes: Relapse: Wasting: Sustained recovery

Relapse after treatment for acute malnutrition is a serious problem facing the humanitarian and development communities. A few studies that have followed up children after initial recovery from moderate acute malnutrition (MAM) have shown that such a relapse is common ${ }^{(1-3)}$. For example, previous research in Malawi found that only $51-63 \%$ of children sustain recovery for 12 months after initial treatment for MAM $^{(1,3)}$. Common childhood illnesses, such as fever, cough, malaria and diarrhoea, are prevalent among those who relapse or die after initial MAM recovery $^{(1,4-6)}$. Mid-upper-arm circumference (MUAC) and weight-for-height $z$ score (WHZ) at enrolment and discharge from a supplementary feeding programme (SFP) have shown to be predictive of relapse during the following year, although duration of treatment was not ${ }^{(7)}$. A recent study from Burkina Faso that included $90 \%$ of children with MAM and $10 \%$ with SAM reported factors with relapse to be low MUAC at discharge, low oil/fat consumption during the follow-up period and incomplete vaccination $^{(6)}$. Among children with SAM, a study in Malawi identified children with HIV to have a higher risk of relapse after treatment ${ }^{(8)}$. Currently, there is no standardised definition of relapse, and the varying rates that have been identified may be because of different follow-up time periods and methodologies ${ }^{(1-5,9)}$. These studies have begun to shed light on factors associated with relapse, yet large gaps in knowledge remain.

It is plausible that returning to the same household $(\mathrm{HH})$ environment that may have contributed to the initial

Abbreviations: cRCT, cluster randomised controlled trial; HFIAS, Household Food Insecurity Access Scale; HH, household; IYCF, infant and young child feeding; MAM, moderate acute malnutrition; MUAC, mid-upper-arm circumference; SES, socio-economic status; SFP, supplementary feeding programme; WASH, water, sanitation and hygiene.

* Corresponding author: H. C. Stobaugh, fax +919 485-5555, email HStobaugh@rti.org 
development of MAM may predispose children to relapse. Infant and young child feeding (IYCF) practices ${ }^{(10-13)}$, unsanitary living conditions ${ }^{(14,15)}$, poor food security and dietary diversity $^{(16,17)}$ and impoverished socio-economic status $(\mathrm{SES})^{(18,19)}$ have all been linked to developing acute malnutrition in various contexts. However, prior studies have not specifically correlated these factors with relapse following initial recovery from MAM. Identifying $\mathrm{HH}$ risk factors associated with relapse could have significant implications for how best to prevent relapse and improve the sustainability of MAM treatment. To identify such factors, we conducted an in-depth $\mathrm{HH}$ survey among children who were followed up for 1 year after recovery from MAM.

\section{Methods \\ Study design and objective}

This observational study consisted of an in-depth HH survey administered prospectively at the time of SFP discharge to a sub-sample of participants within a larger cluster randomised controlled trial (cRCT). Complete methods of the parent cRCT have previously been described in detail ${ }^{(3)}$. In brief, children aged 6-62 months who had recovered from MAM, defined as MUAC $\geq 12.5 \mathrm{~cm}$ without bipedal oedema ${ }^{(20)}$, were recruited from rural health clinics in southern Malawi. In all, eleven clinics were randomly assigned to receive nutrition counselling in addition to a package of health and nutrition interventions at the time of SFP discharge, whereas ten clinics were randomly selected to receive nutrition counselling only. Children were followed up for 12 months after discharge to assess the impact of the intervention package on sustained recovery.

The aim of this study was to identify $\mathrm{HH}$ factors that may be associated with sustained recovery, including SES, dietary diversity, food insecurity, child care practices and water, sanitation and hygiene (WASH). The HH survey was conducted only among children in the control group in the parent $\mathrm{CRCT}^{(3)}$.

\section{Study setting}

The study was conducted in five districts (Chikwawa, Chiradzulu, Machinga, Mulanji and Zomba Districts) across the Southern Region in Malawi where most of the population consists of subsistence farmers. Conditions are often unsanitary, without access to clean drinking water, and $\mathrm{HH}$ are often at far distances from functioning health clinics. Child malnutrition is widespread in Malawi; over $42 \%$ of children under 5 years old are stunted and $17 \%$ are underweight ${ }^{(21)}$.

\section{Subject participation}

Children were enrolled in the study at the time of discharge from an SFP. These children were the last 315 children enrolled in all control sites from the parent cRCT study. Upon enrolment, caregivers scheduled an appointment for a home visit within a week of the child's discharge from the SFP. The survey was administered at the child's home by a trained data collector (either a senior paediatric research nurse or a community health worker) and lasted approximately $1 \mathrm{~h}$. Caregivers were asked to return to the clinic for subsequent follow-up visits at 1, 3, 6 and 12 months after enrolment to reassess the child's nutrition status and clinical signs of illness, including diarrhoea and fever. HH food security was also assessed at each follow-up visit using the validated, nineitem Household Food Insecurity Access Scale (HFIAS) ${ }^{(22)}$.

\section{Household survey}

Data collected from the HH survey consisted of information pertaining to (1) SES; (2) IYCF practices; (3) HH food security; (4) WASH; and (5) maternal perceptions of MAM, the SFP and relapse following MAM.

Socio-economic status. We collected indicators based on an SES index by Psaki et $a l .^{(23)}$, recently validated in an eightcountry study. This index, called the WAMI index, comprised four main components - water and sanitation, assets, maternal education and $\mathrm{HH}$ income. WAMI was chosen because of its simplicity for field use and association with height-for-age $z$ score $^{(24)}$ in childhood ${ }^{(23)}$. However, in this study, monthly $\mathrm{HH}$ income was not surveyed because many of the families in this context have only informal avenues of widely fluctuating monthly income $^{(19)}$. In addition, the water and sanitation component was not included to avoid redundancy with other WASH indicators elsewhere in the final model. To account for these deviations, we collected information on additional assets and livestock to help distinguish different levels of wealth within the local context. After conducting a principal component analysis, we determined that a single SES measure was not appropriate for this data set because principal component analysis showed lack of correlation between indicators. Therefore, rather than using one overall SES index, we included the following individual SES-related variables in the final regression model: maternal education, number of $\mathrm{HH}$ assets (out of eleven total), number of rooms in a house and ownership of any livestock. Table 1 contains information on the definition and scoring of each SES indicator.

Infant and young child feeding practices. Indicators included in the $\mathrm{HH}$ survey regarding IYCF practices were based on current international recommendations ${ }^{(25,26)}$. Indicators included (1) breast-feeding practices, (2) the introduction of complementary foods, (3) minimum dietary diversity and (4) minimum meal frequency. Because recommended feeding practices vary by age ${ }^{(13,25,26)}$, information used in the breastfeeding practices and minimum meal frequency indicators differed according to the age of the child. Table 2 contains information on the definition and scoring of each IYCF practice indicator.

Food security. HH food security was assessed using HFIAS ${ }^{(22)}$, a series of nine questions regarding the food security situation at the $\mathrm{HH}$ level. The HFIAS has been validated in numerous countries around the world ${ }^{(20)}$. Scores range from 0 to 27 , and higher scores represent increased food insecurity, whereas lower scores represent better food security. HFIAS was administered to the caregiver at the time of admission into the SFP, as well as 1, 3, 6 and 12 months after SFP discharge. Scores from all time points throughout the year were averaged. Food 
Table 1. Socio-economic status indicators from the household survey

\begin{tabular}{ll}
\hline Indicators & Definition \\
\hline Maternal education & $\begin{array}{c}\text { Number of years of completed education, ranging from no education up to 'Form 4, the completion of 12 years of } \\
\text { primary and secondary education in Malawi. (No respondents completed any education beyond Form 4) } \\
\text { Ossets from the following list owned by anyone in the home: mattress, bicycle, chair or bench, radio, mobile phone, } \\
\text { flashlight, cabinet, pair of shoes, candle, lantern and bank account }\end{array}$ \\
Number of rooms in house & $\begin{array}{l}\text { Number of separate rooms in a house, defined as a physical wall, which did not include sheets or curtains dividing } \\
\text { a space. The minimum and maximum number of rooms reported were 1 and 6, respectively } \\
\text { Ownership of livestock }\end{array} \quad \begin{array}{c}\text { If anyone in the home owned any livestock (chickens, goats, cattle, dogs, pigs, guinea fowl), this was considered } \\
\text { an asset rather than a separate livelihood given the fact that all participants were subsistence farmers and the } \\
\text { ownership of animals would be indicative of additional wealth }\end{array}$ \\
\hline
\end{tabular}

Table 2. Infant and young child feeding practices indicators from the household survey

\begin{tabular}{|c|c|c|}
\hline Indicators & Definition & Score \\
\hline Continued breast-feeding & $\begin{array}{l}\text { For children under } 24 \text { months of age, the score was } 1 \text { if they were currently breast-feeding. For children over } \\
24 \text { months, the score was } 1 \text { if the age at which they stopped breast-feeding was beyond } 24 \text { months }\end{array}$ & 0 or 1 \\
\hline $\begin{array}{l}\text { Introduction of } \\
\text { complementary foods }\end{array}$ & The score was 1 if solid and/or semi-solid foods were introduced at $6-8$ months of age & 0 or 1 \\
\hline Minimum dietary diversity & $\begin{array}{l}\text { The score was } 1 \text { if they consumed four or more food groups (among grains, legumes, meats, eggs, vitamin-A-rich } \\
\text { fruit and vegetables, other fruit and vegetables, and dairy products) based on 24-h dietary recall } 25,26)\end{array}$ & 0 or 1 \\
\hline Minimum meal frequency & $\begin{array}{l}\text { The score was } 1 \text { if they achieved minimum meal frequency in the previous } 24 \mathrm{~h} \text {. Minimum frequency is } 2 \text { or more } \\
\text { meals for ages } 6 \text { to }<9 \text { months, } 3 \text { or more meals for ages } 9 \text { to }<12 \text { months and } 4 \text { or more meals for ages } \\
12 \text { months and older }{ }^{(12)}\end{array}$ & 0 or 1 \\
\hline
\end{tabular}

Table 3. Water, sanitation and hygiene indicators from the household $(\mathrm{HH})$ survey

\begin{tabular}{|c|c|c|}
\hline Indicators & Definition and scoring & Score \\
\hline Cleanliness of caregiver's hands & $\begin{array}{l}\text { The score was } 1 \text { if the caregiver's hands were observed to have a 'clean appearance', and } 0 \text { if 'the } \\
\text { presence of visible dirt' or 'a dirty appearance' was observed }\end{array}$ & 0 or 1 \\
\hline Cleanliness of child's hands & $\begin{array}{l}\text { The score was } 1 \text { if the child's hands were observed to have a 'clean appearance' or } 0 \text { if 'the presence of } \\
\text { visible dirt' or 'a dirty appearance' was observed }\end{array}$ & 0 or 1 \\
\hline Improved water source & $\begin{array}{l}\text { The score was } 1 \text { if all water sources were improved sources of drinking water (piped water into dwelling, } \\
\text { piped water into yard/plot, public tap or standpipe, tube well or borehole, protected dug well, protected } \\
\text { spring and rainwater) }\end{array}$ & 0 or 1 \\
\hline Lids on water storage containers & The score was 1 if all water storage containers were observed to have lids ${ }^{(35)}$ & 0 or 1 \\
\hline Treat drinking water & $\begin{array}{l}\text { The score was } 1 \text { if action was taken to make the drinking water safe for consumption (boiling, bleaching, } \\
\text { adding } \mathrm{Cl} \text {, straining through a cloth, use of water filer, solar disinfection and let it stand and settle) }\end{array}$ & 0 or 1 \\
\hline Hand-washing & The score was 1 if soap or ash was used during a hand-washing demonstration ${ }^{(27,30)}$ & 0 or 1 \\
\hline $\begin{array}{l}\text { Knowledge of critical times for } \\
\text { caregiver hand-washing }\end{array}$ & $\begin{array}{l}\text { The score was } 1 \text { for listing all five critical time points for hand-washing (after defecation, after cleaning a } \\
\text { child, before preparing food, before feeding a child, before eating), as defined by UNICEF }(30,33)\end{array}$ & 0 or 1 \\
\hline Frequency of bathing the child & The score was 1 if the child was bathed at least once per day during the previous week w $^{(34)}$ & 0 or 1 \\
\hline Improved sanitation facility & $\begin{array}{l}\text { The score was } 1 \text { if } \mathrm{HH} \text { members used an improved sanitation facility (flush toilet, piped sewer system, } \\
\text { septic tank, flush/pour flush to pit latrine, ventilated improved pit latrine or pit latrine with slab) }{ }^{(29)}\end{array}$ & 0 or 1 \\
\hline
\end{tabular}

security fluctuates throughout the year between times when food availability is higher just after the single annual harvest and times when food is scarcer before harvest. An arithmetic average over the course of the year was chosen to help account for these fluctuations.

Water, sanitation and hygiene. WASH indicators used by international agencies ${ }^{(27-29)}$ and the 2011 Demographic and Health Survey (DHS) ${ }^{(30)}$ were included to capture WASH conditions and practices among participating $\mathrm{HH}$. Given prior associations between cleanliness of hands and child health outcomes ${ }^{(31,32)}$, the caregiver's hands and the child's hands were visually inspected for cleanliness, which included a threepoint scale denoting 'clean,' 'no visible dirt but unclean appearance' and 'visible dirt' regarding the palms, finger pads and finger nails (later combined into two categories of clean and unclean $)^{(27)}$. During data collection training, data collectors were shown several examples of proper scoring, including tests of intra- and inter-rater reliability. This training included large group demonstrations and small group exercises. A final test was administered at the end of the training to ensure that proper scoring was demonstrated.

Use of improved water sources and use of sanitation facilities were based on the WHO definitions ${ }^{(29,30)}$. Respondents were asked if they take action to treat water, according to the DHS format ${ }^{(30)}$. Water storage containers were assessed for having fitted lids ${ }^{(33)}$. Hygiene was assessed by direct observation of whether a caregiver used soap during a hand-washing demonstration ${ }^{(27,28)}$, knowledge regarding five critical times for hand washing ${ }^{(28,30,33)}$ and the frequency with which the child is bathed $^{(34)}$. Table 3 contains information on the definition and scoring of each WASH indicator. 


\section{Outcome definitions}

At the end of the 12-month follow-up period, each child was classified as having 'sustained recovery', defined as having MUAC $\geq 12.5 \mathrm{~cm}$ at every follow-up visit for 12 months; 'relapsed to MAM', defined as MUAC $<12.5 \mathrm{~cm}$ and $\geq 11.5 \mathrm{~cm}$ at any point during the follow-up period; 'developed SAM', defined as MUAC $<11.5 \mathrm{~cm}$ and/or bipedal oedema (kwashiorkor) at any point during the follow-up period; 'died'; or 'lost to follow-up,' defined as defaulting on a scheduled visit and never returning. Poor outcomes were considered to be relapsing to MAM, developing SAM, being lost to follow-up or dying. If a child experienced two such outcomes over the course of the follow-up period, the more severe category was assigned as the final outcome.

To date, there is no consensus on the definition of relapse after recovery from MAM. Our definition of relapse and the time period in which we followed up children was chosen in order to compare results with previous studies that measured relapse similarly $^{(1,3)}$. Furthermore, in order to better understand the nutritional status following initial recovery from MAM, we chose to err on the side of inclusion, rather than exclusion, for our final definition of relapse. Therefore, we chose a 12-month follow-up time period so as to capture both children who relapse in the first few months following discharge and also those who relapse repeatedly over the course of a year. Following up children for a full year also allowed us to account for seasonality in all study participants. A relapse during the hungry season that occurs several months after initial recovery from MAM could be perceived as a 'new episode' of MAM, given the increased likelihood of malnutrition during this season of high food insecurity. However, it has been demonstrated that children who recover from acute malnutrition (either SAM or MAM) are at a higher risk of relapse, morbidity and mortality than those who were not previously malnourished ${ }^{(1,36)}$. Therefore, because a previously malnourished child is at a higher risk than a non-previously malnourished children, we defined additional malnutrition during (or not during) the hungry season within the follow-up period as a relapse rather than a new episode.

\section{Ethical approval}

The study was conducted according to the guidelines laid down in the Declaration of Helsinki, and all procedures involving human subjects were approved by the University of Malawi's College of Medicine Research and Ethics Committee (P. 11/12/ 1311), Washington University's Human Research Protection Office (ID: 201301124) and Tufts University's Health Sciences Campus Institutional Review Board (IRB\#: 11887). Permission to conduct the study was obtained by each site's District Health Officer and/or District Nutritionist. Written informed consent was obtained from all caregivers.

\section{Statistical analyses}

Sample size was determined by the feasibility of recruiting children in the time frame during April to June 2015 of enrolment into the larger cRCT. Recruitment was limited to this time period as the inception of the study idea began after the larger cRCT was underway; therefore, the largest number of participants were recruited after ethical approval was obtained and before the close of the larger study. Given the total sample size of 312 and to achieve a statistical power of $80 \%$ and a two-sided significance level of $0 \cdot 05$, minimally detectable effect sizes were calculated retrospectively to be a 12 to 16 percentage point difference between proportions for binary variables and a mean difference of $0 \cdot 9,0 \cdot 3,0 \cdot 4,0 \cdot 4,1 \cdot 1$ and 1.2 for years of education completed by the caregiver, number of rooms in the house, meal frequency, dietary diversity score, average HFIAS score and number of times the child was bathed during the previous week, respectively.

All data were double-entered into an Access (Microsoft Corp.) database and verified against original forms when discrepancies were identified. To compare individual indicators between $\mathrm{HH}$ with children who sustained recovery and those who did not, bivariate analyses were conducted using Student's $t$ test for continuous variables and chi-squared for binary variables with adjustment for clustering at the health clinic level. $P$ values $<0.05$ were considered to be statistically significant.

Binary logistic regression was used to identify the indicators associated with sustained recovery while accounting for other factors. Cluster-adjusted robust standard errors were used to account for the clustering at the health clinic level. Variables used in the full model included sex; age at the time of admission to SFP; whether the child had fever during the 2 weeks before admission into the SFP; whether the child had diarrhoea during the 2 weeks before admission into the SFP; discharge MUAC; discharge WHZ score; years of education completed by the caregiver; number of rooms in the house; whether any livestock was owned; number of $\mathrm{HH}$ assets owned (out of eleven); whether the child was currently breast-feeding at the time of SFP discharge (for children under 24 months) or whether the child continued to breast-feed up to the age of 24 months (for children over 24 months); minimum dietary diversity; minimum meal frequency; average HFAIS score from all followup visits; whether all of the drinking water was retrieved from improved water sources; use of an improved sanitation facility; cleanliness of caregiver's hands; cleanliness of child's hands; and whether the child was bathed daily during the previous week. All statistical analyses were conducted using Stata Version 13.0 (StataCorp LP).

\section{Results}

Out of the 1487 children included in the parent cRCT, a total of 315 children were enrolled in this study and completed the in-depth HH survey. Three surveys were excluded because of uninterpretable data, leaving a total of 312 for final analysis. The proportion of children who sustained recovery for the duration of the 12-month follow-up period after initial recovery from MAM was $58 \%$.

In bivariate analysis comparing $\mathrm{HH}$ indicators between those who sustained recovery and those who did not, few individual indicators differed (Table 4 and online Supplementary Table S1). A larger proportion of $\mathrm{HH}$ with a child who sustained recovery had lids on all water storage containers than those $\mathrm{HH}$ whose child did not sustain recovery (Table 4). Although not statistically significant, caregivers' hands were observed by 
Table 4. Comparison of indicators regarding household (HH) characteristics: socio-economic status (SES); infant and young child feeding (IYCF) practices; water, sanitation and hygiene (WASH) conditions; and maternal perceptions between children who sustained recovery and those who did not sustain recovery following treatment for moderate acute malnutrition (MAM) in the supplementary feeding programme (SFP)

(Numbers and percentages; mean values and standard deviations)

\begin{tabular}{|c|c|c|c|c|c|c|c|c|c|}
\hline \multirow[b]{2}{*}{ Characteristic $^{*}$} & \multicolumn{4}{|c|}{ Sustained recovery $(n 180)$} & \multicolumn{5}{|c|}{ Did not sustain recovery $(n$ 132) } \\
\hline & $n$ & $\%$ & Mean & SD & $n$ & $\%$ & Mean & SD & $P \dagger$ \\
\hline \multicolumn{10}{|l|}{$\mathrm{HH}$ characteristics and SES } \\
\hline Other children in home ever had acute malnutrition & 51 & 29 & & & 32 & 25 & & & 0.419 \\
\hline Years of education completed by the caregiver & & & 3.89 & $2 \cdot 75$ & & & $4 \cdot 17$ & 2.69 & 0.393 \\
\hline Number of rooms in the house & & & 1.87 & 0.96 & & & 1.95 & 1.02 & 0.533 \\
\hline Ownership of any livestock & 80 & 45 & & & 68 & 53 & & & 0.501 \\
\hline Number of $\mathrm{HH}$ assets owned (out of 11 total) & & & $3 \cdot 13$ & 1.58 & & & $3 \cdot 13$ & 1.64 & 0.999 \\
\hline \multicolumn{10}{|l|}{ IYCF } \\
\hline Continued breast-feeding & 127 & 75 & & & 103 & 82 & & & 0.341 \\
\hline Appropriate timing for introduction of solid food & 145 & 81 & & & 96 & 74 & & & 0.167 \\
\hline Minimum meal frequency & 69 & 39 & & & 55 & 42 & & & 0.641 \\
\hline Minimum dietary diversity score & 112 & 62 & & & 77 & 58 & & & 0.794 \\
\hline \multicolumn{10}{|l|}{ Food security } \\
\hline Average HFIAS score throughout 1 yearł & & & 12.55 & 3.44 & & & $12 \cdot 88$ & 3.54 & 0.831 \\
\hline \multicolumn{10}{|l|}{ WASH } \\
\hline Caregiver's hands observed to be 'clean' ( $0=$ 'visible dirt' or 'dirty appearance', $1=$ 'clean') & 66 & 37 & & & 32 & 24 & & & 0.117 \\
\hline Child's hands observed to be 'clean' ( $0=$ 'visible dirt' or 'dirty appearance', $1=$ 'clean') & 78 & 43 & & & 55 & 42 & & & 0.866 \\
\hline Uses improved source for drinking water & 134 & 74 & & & 90 & 46 & & & 0.225 \\
\hline All water storage containers have lids & 78 & 44 & & & 40 & 32 & & & 0.029 \\
\hline Takes action to make drinking water safer & 93 & 52 & & & 73 & 55 & & & 0.523 \\
\hline Used soap or ash during hand-washing demonstration & 46 & 26 & & & 30 & 24 & & & 0.788 \\
\hline Knowledge of all five critical times for hand-washing & 16 & 9 & & & 14 & 11 & & & 0.611 \\
\hline Child bathed at least once per day during the previous week & 154 & 86 & & & 105 & 81 & & & 0.675 \\
\hline Uses improved sanitation facility & 27 & 15 & & & 11 & 8 & & & 0.077 \\
\hline \multicolumn{10}{|l|}{ Caregiver perceptions of SFP, MAM and relapse } \\
\hline Referral source for attending SFP & & & & & & & & & 0.956 \\
\hline Health professional or $\mathrm{CHW}$ & 141 & 79 & & & 105 & 80 & & & 0.868 \\
\hline Self & 33 & 18 & & & 24 & 18 & & & 0.954 \\
\hline Friends or neighbours & 5 & 3 & & & 3 & 2 & & & 0.774 \\
\hline \multicolumn{10}{|l|}{ Reason caregiver brought the child to the SFP site } \\
\hline Routine check-up & 20 & 23 & & & 7 & 13 & & & 0.448 \\
\hline Influenced by friends or neighbours & 2 & 2 & & & 1 & 2 & & & 0.870 \\
\hline Child seemed ill (fever, cough or diarrhoea) & 42 & 48 & & & 25 & 47 & & & 0.944 \\
\hline Child seemed malnourished (appeared thin) & 17 & 20 & & & 16 & 30 & & & 0.345 \\
\hline Child experienced a lack of appetite & 2 & 2 & & & 0 & 0 & & & 0.488 \\
\hline Due to referral & 4 & 5 & & & 4 & 8 & & & 0.465 \\
\hline Perceived child to be sick upon SFP admission & 86 & 53 & & & 64 & 54 & & & 0.908 \\
\hline Perceived child to be malnourished upon SFP admission & 112 & 64 & & & 81 & 64 & & & 0.959 \\
\hline Perceived child's status to improve during treatment & 180 & 100 & & & 132 & 100 & & & $\mathrm{n} / \mathrm{a}$ \\
\hline Understood that relapse was possible & 76 & 42 & & & 55 & 42 & & & 0.888 \\
\hline Understood that re-enrolment to SFP was possible & 154 & 88 & & & 113 & 86 & & & 0.537 \\
\hline Thought actions could be taken to prevent MAM & 150 & 84 & & & 110 & 84 & & & 0.943 \\
\hline
\end{tabular}

HFIAS, Household Food Insecurity Access Scale (0-27); CHW, community health worker.

* Missing values ranged from 0 to 7 for all indicators with the exception of perceived child to be malnourished upon SFP admission; used soap or ash during hand-washing demonstration; continued breast-feeding; and perceived the child to be sick upon SFP admission, which had 11, 13, 15 and 31 missing values, respectively.

$\dagger P$ values derived using Student's $t$ test or $x^{2}$ with adjustment for clustering. Minimally detectable effect sizes were calculated retrospectively for proportions to be a 12 to 16 percentage point difference for all main binary variables and a mean difference of $0.9,0.3,0.4,0.4,1.1$ and 1.2 for years of education completed by the caregiver, number of rooms in the house, meal frequency, dietary diversity score, average HFIAS score and number of times the child was bathed during the previous week, respectively. For example, we have $80 \%$ power to detect a mean HFIAS difference of 1.1 . Although the mean difference in HFIAS score is $<1.1$ (at $0.33 \mathrm{HFIAS}$ score), as seen here, this translates to extremely similar $\mathrm{HH}$ food insecurity situations between the two groups and we can confidently conclude no statistical or practical difference.

$\ddagger$ HFIAS ranging from 0 to 27 was assessed at 1, 3, 6 and 12 months of follow-up. These scores were averaged to provide the food security situation at the household level at multiple points throughout the year. Changes in participants' HFIAS scores between visits ranged from 0 to 23 and averaged a ten-point difference between the lowest and highest HFIAS scores across follow-up visits.

enumerators to be cleaner among children who sustained recovery. In addition, the proportions of $\mathrm{HH}$ that used an improved sanitation facility or improved sources for drinking water were nearly twice as high among the sustained recovery group compared with those who failed to sustain recovery (although without statistical significance). No differences were found between the two groups regarding SES indicators; IYCF practices; food security; or maternal perceptions of SFP, MAM and relapse.
Similar results were found when controlling for other factors in binary logistic regression (Table 5). HH that had fitted lids on all water storage containers were more likely to have a child who sustained recovery than those who did not have lids on all storage containers. Caregivers whose hands appeared to be clean and $\mathrm{HH}$ that used an improved sanitation facility also showed strong trends to having a child sustain recovery. $\mathrm{HH}$ SES, food security and IYCF practices were not significantly associated with whether a child sustained recovery. Children 
Table 5. Factors associated with children who sustained recovery for 12 months following recovery from moderate acute malnutrition in multivariate logistic regression model*

(Adjusted odds ratios and $95 \%$ confidence intervals)

\begin{tabular}{|c|c|c|c|}
\hline Independent variables $†$ & Adjusted OR & $95 \% \mathrm{Cl}$ & $P$ \\
\hline Age (months) & 1.01 & $0.98,1.05$ & 0.482 \\
\hline Female & 1.61 & $0.97,2.66$ & 0.066 \\
\hline MUAC at SFP discharge $(\mathrm{mm})$ & 1.20 & $1.09,1.31$ & $<0.001$ \\
\hline WHZ at SFP discharge & 1.12 & $0.55,1.11$ & 0.745 \\
\hline Fever in 2 weeks before SFP admission & 0.78 & $0.41,1.49$ & 0.456 \\
\hline Diarrhoea in 2 weeks before SFP admission & 1.52 & $0.70,3.30$ & 0.291 \\
\hline Years of education completed by caregiver & 0.94 & $0.86,1.02$ & 0.137 \\
\hline Number of rooms in the house & 0.98 & $0.76,1.27$ & 0.881 \\
\hline Ownership of any livestock & 0.78 & $0.55,1.11$ & 0.170 \\
\hline Number of $\mathrm{HH}$ assets owned (out of 11 total) & 1.02 & $0.85,1.23$ & 0.834 \\
\hline Continued breast-feeding & 1.04 & $0.36,2.99$ & 0.941 \\
\hline Appropriate timing for introduction of solid food & 1.86 & $0.89,3.89$ & 0.098 \\
\hline Minimum meal frequency & 0.87 & $0.53,1.43$ & 0.577 \\
\hline Minimum dietary diversity & 0.95 & $0.51,1.77$ & 0.880 \\
\hline Average HFIAS score & 0.98 & $0.88,1.09$ & 0.680 \\
\hline Uses improved source for drinking water & 1.45 & $0.66,3.18$ & 0.353 \\
\hline All water storage containers have lids & 1.79 & $1.20,2.68$ & 0.004 \\
\hline Uses improved sanitation facility & 1.46 & $0.95,2.26$ & 0.083 \\
\hline Used soap or ash during hand-washing demonstration & 0.84 & $0.29,2.37$ & 0.736 \\
\hline Caregiver's hands appear 'clean' & 2.47 & $0.99,6 \cdot 18$ & 0.053 \\
\hline Child's hands appear 'clean' & 0.65 & $0.31,1.33$ & 0.238 \\
\hline Child bathed at least once per day during the previous week & 1.28 & $0.67,2.42$ & 0.455 \\
\hline
\end{tabular}

MUAC, mid-upper-arm circumference; SFP, supplementary feeding programme; WHZ, weight-for-height $z$ score; HH, Household; HFIAS, Household Food Insecurity Access Scale.

* Logistic regression model included cluster robust standard errors to account for clustering at the health clinic level.

† See Tables 1-3 for more details on definitions of indicators.

with a higher MUAC upon discharge from initial MAM treatment in an SFP were more likely to sustain recovery than those with a lower discharge MUAC.

\section{Discussion}

These results show that improved conditions associated with WASH, including $\mathrm{HH}$ having fitted lids on water storage containers, caregivers having clean hands and $\mathrm{HH}$ using an improved sanitation facility, were each associated with sustained recovery after MAM. Interestingly, factors related to SES, food security and IYCF practices were not significant. Given these results, we hypothesise that improved WASH conditions in tandem with management of MAM through supplemental feeding programmes have the possibility to decrease relapse following recovery from MAM.

Although many studies have been conducted regarding the impact of WASH on child health outcomes, such as diarrhoea $^{(37)}$ and helminth infection ${ }^{(38)}$, fewer and less rigorous studies have been used regarding nutritional outcomes and show little evidence of impact. A recent Cochrane review identified five cRCT that measured the effect of WASH interventions on nutritional status and found no evidence that WASH interventions have an impact on weight-for-age $z$ score or WHZ and only a small effect on height-for-age $z$ score $^{(24,39)}$. That said, the interventions were short in duration; no study considered the effect of a complete package of WASH interventions; and no study examined the impact on relapse rates following initial recovery from MAM. A prospective, randomised clinical trial that examines the impact of a package of
WASH interventions on relapse among children following recovery from MAM or SAM is warranted.

Further studies are needed to generate a better understanding of the potential causal pathways between WASH conditions and sustained recovery from MAM. These include in-depth biological analyses regarding diarrhoeal diseases, intestinal parasite infections and environmental enteric dysfunction (EED) during and following the state of moderate malnutrition. A recent study shows a high proportion of subclinical inflammation in children at the time of MAM diagnosis ${ }^{(40)}$, a likely sign of EED. Diarrhoea and intestinal parasites have been shown to exacerbate acute malnutrition ${ }^{(41-44)}$ and, if gone untreated at the time of discharge from SFP, could leave children susceptible to relapse. However, results from the larger CRCT, in which this current study was embedded, found that providing a single dose of deworming medication and a 14-d course of Zn supplementation at the time of SFP discharge (as part of a larger package of services) did not improve relapse-free survival curves for 12 months following recovery from $\mathrm{MAM}^{(3)}$.

Although evidence from this study points to the potential for improved HH WASH conditions to reduce relapse rates following recovery from MAM, improved $\mathrm{HH}$ WASH condition is likely only one of many factors that must be explored to improve MAM treatment so that recovery is better sustained. For example, our study shows that a higher MUAC at SFP discharge is predictive of sustaining recovery during the following year. Higher WHZ and MUAC at the time of admission and discharge from MAM treatment have also been observed in other studies to be more predictive of sustained recovery than a longer duration of treatment ${ }^{(1,7)}$. Therefore, the combination of improved WASH condition, as well as improved anthropometrics 
at the time of SFP discharge, may have a significant impact on reducing relapse rates following SFP.

There are several limitations of the study design to be considered. First, the lack of variability in the results is reflective of a relatively homogeneous study population. The indicators chosen in the study may not have been specific enough to this study population to identify true variations that did exist. Second, although the WASH indicators included in the HH survey are standard indicators commonly used for assessments and programme evaluations, they do not fully capture all aspects of WASH conditions. For example, although a $\mathrm{HH}$ may have access to an improved sanitation facility, other members in the community may still practice open defecation, which reduces the positive effects of that individual HH's use of the improved sanitation facility. Third, the sampling of the study population was based on convenience at the end of the study, as opposed to a random selection throughout the duration of the larger CRCT in which this sub-study was embedded. This may have led to some biases in the selection of the study population.

With the exception of a few WASH indicators, our results show that the vast majority of $\mathrm{HH}$ characteristics and caregiving practices in this context did not greatly differ between $\mathrm{HH}$ whose children relapsed and those who sustained recovery. In this homogeneous low-income population, factors relating to SES, IYCF practices and food security that are commonly associated with malnutrition $^{(11-14,17-20)}$ do not distinguish between children who sustain MAM recovery and those who do not. This suggests that what distinguishes those who relapse and those who do not is related less to $\mathrm{HH}$ conditions and relatively more to individual determinants of a child's health and nutrition. Similar findings were also demonstrated in a recent follow-up study of children after recovery from SAM, whereby clinical and anthropometric indicators were predictive of death during the follow-up period, yet social or economic factors were not ${ }^{(10)}$. Certainly, $\mathrm{HH}$ factors are important to the overall well-being of children, and improvements in the environment and caregiving practices should remain goals in public health interventions and development programmes. Yet, on the basis of these results, increasing nutrition-sensitive programming alone is not likely to reduce markedly the large percentage of children experiencing relapse following MAM. Rather, these programmes should be considered an important complement to improved SFP treatment and follow-up protocols that address risk factors of relapse at the level of the individual child.

\section{Acknowledgements}

We thank the Malawian nurses at the St. Louis Nutrition Project for their dedication to collecting high-quality data throughout the course of this study.

This publication was supported by US Agency for International Development (USAID) (H. C. S., C. T., K. M. M., I. T., M. J. M., Cooperative Agreement/grant no. AID-OAA-A-12-0005); the Children's Discovery Institute of Washington University and St. Louis Children's Hospital (I. T.); and the Office of Food for Peace (FFP) of the Bureau for Democracy, Conflict and Humanitarian Assistance (B. L. R., P. W., I. H. R., Cooperative Agreement no. AID-OAA-C-16-00020). USAID had no role in the design, analysis or writing of this article. The contents are the responsibility of the authors and do not necessarily reflect the views of USAID or the US government, nor does any mention of trade names, commercial products or organisations imply endorsement by USAID or the US government.

H. C. S., B. L. R., P. W., I. H. R., M. J. M. and I. T. formulated the research and designed the study. H. C. S. and I. T. enrolled participants and conducted the study. C. T., K. M. M., M. J. M. and I. T. supervised the implementation of the study and all study protocols. H. C. S. cleaned and analysed the data, wrote the first draft of the manuscript and has primary responsibility for the paper's final content. All authors edited the manuscript and approved its final contents.

The authors declare that there are no conflicts of interest. This manuscript does not include animal studies, in vitro studies or probiotics.

\section{Supplementary material}

For supplementary material/s referred to in this article, please visit https://doi.org/10.1017/S0007114518000363

\section{References}

1. Chang C, Trehan I, Wang R, et al. (2013) Children successfully treated for moderate acute malnutrition remain at risk for malnutrition and death in the subsequent year after recovery. J Nutr 143, 215-220.

2. Nackers F, Broillet F, Oumarou D, et al. (2010) Effectiveness of ready-to-use therapeutic food compared to a corn/soyblend-based pre-mix for the treatment of childhood moderate acute malnutrition in Niger. J Trop Pediatr 56, 407-413.

3. Stobaugh HC, Bollinger LB, Adams SE, et al. (2017) Effect of a package of health and nutrition services on sustained recovery in children after moderate acute malnutrition and factors related to sustaining recovery: a cluster-randomized trial. $\mathrm{AmJ}$ Clin Nutr 106, 657-666.

4. Ashraf H, Alam NH, Chisti MJ, et al. (2012) A follow-up experience of 6 months after treatment of children with severe acute malnutrition in Dhaka, Bangladesh. J Trop Pediatr 58, 4 .

5. Begashaw M (2013) SQUEAC report on Baure and SLEAC on Baure, Dutsi, Daura and Zango, Katsina LGA, Nigeria. New York: Coverage Monitoring Network.

6. Somasse YE, Dramaix M, Bahwere P, et al. (2016) Relapses from acute malnutrition and related factors in a communitybased management programme in Burkina Faso. Matern Child Nutr 12, 908-917.

7. Trehan I, Banerjee S, Murray E, et al. (2015) Extending supplementary feeding for children younger than 5 years with moderate acute malnutrition leads to lower relapse rates. J Pediatr Gastroenterol Nutr 60, 544-549.

8. Kerac M, Bunn J, Chagaluka G, et al. (2014) Follow-up of postdischarge growth and mortality after Treatment for Severe Acute Malnutrition (FuSAM Study): a prospective cohort study. PLOS ONE 9, e96030.

9. Somasse YE, Dramaix M, Bahwere P, et al. (2015) Relapses from acute malnutrition and related factors in a communitybased management programme in Burkina Faso. Matern Child Nutr 12, 908-917.

10. Khatoon T, Mollah MAH, Choudhury AM, et al. (2011) Association between infant and child-feeding index and nutritional status: Results from a cross-sectional study among children 
attending an urban hospital in Bangladesh. J Health Popul Nutr 29, 349-356.

11. Ma J-Q, Zhou L-L, Hu Y-Q, et al. (2012) A summary index of infant and child feeding practices is associated with child growth in urban Shanghai. BMC Public Health 12, 568.

12. Ruel MT \& Menon P (2002) Child feeding practices are associated with child nutritional status in Latin America: innovative uses of the demographic and health surveys. $J$ Nutr $\mathbf{1 3 2}$, 1180-1187.

13. Sawadogo PS, Martin-Prével Y, Savy M, et al. (2006) An infant and child feeding index is associated with the nutritional status of 6- to 23-month-old children in rural Burkina Faso. J Nutr 136, 656-663.

14. Humphrey JH (2009) Child undernutrition, tropical enteropathy, toilets, and handwashing. Lancet 374, 1032-1035.

15. Ngure FM, Reid BM, Humphrey JH, et al. (2014) Water, sanitation, and hygiene (WASH), environmental enteropathy, nutrition, and early child development: making the links. Ann N Y Acad Sci 1308, 118-128.

16. Kennedy GL, Pedro MR, Seghieri C, et al. (2007) Dietary diversity score is a useful indicator of micronutrient intake in non-breast-feeding Filipino children. J Nutr 137, 472-477.

17. Moursi MM, Arimond M, Dewey KG, et al. (2008) Dietary diversity is a good predictor of the micronutrient density of the diet of 6- to 23-month-old children in Madagascar. J Nutr 138, 2448-2453.

18. Nandy S, Irving M, Gordon D, et al. (2005) Poverty, child undernutrition and morbidity: new evidence from India. Bull World Health Organ 83, 210-216.

19. O'Donnell O, van Doorslear E, Wagstaff A, et al. (2007) Analyzing Health Equity Using Household Survey Data: A Guide to Techniques and Their Implementation. Washington, DC: World Development Institute, World Bank.

20. World Health Organization (2010) WHO, UNICEF, WFP, UNHCR Consultation on the Programmatic Aspects of the Management of Moderate Malnutrition in Children Under Five Years of Age. Geneva: WHO.

21. World Health Organization (2017) World Health Statistics 2017: Monitoring Health for the SDGs, Sustainable Development Goals. Geneva: WHO.

22. Coates J, Swindale A \& Bilinsky P (2007) Household Food Insecurity Access Scale (HFIAS) for Measurement of Household Food Access: Indicator Guide. Washington, DC: USAID.

23. Psaki S, Seidman JC, Miller M, et al. (2014) Measuring socioeconomic status in multicountry studies: results from the either-country MAL-ED study. Popul Health Metr 12, 8.

24. Victora CG, Requejo JH, Barros AJ, et al. (2016) Countdown to 2015: a decade of tracking progress for maternal, newborn, and child survival. Lancet 387, 2049-2059.

25. World Health Organization, United Nations Children's Fund \& International Food Policy Research Institute, et al. (2008) Indicators for Assessing Infant and Young Child Feeding Practices; Part 1 Definitions. Geneva: WHO.

26. Food and Nutrition Technical Assistance (2010) Indicators for Assessing Infant and Young Child Feeding Practices: Part 2 Measurement. Washington, DC: World Health Organization.

27. Ram P (2013) Practical Guidance For Measuring Handwashing Behavior: 2013 Update. New York: World Bank Water and Sanitation Program.
28. Vujcic J \& Ram P (2013) Handwashing Promotion Monitoring and Evaluation Module. New York: United Nations Children's Fund.

29. World Health Organization \&United Nations Children's Fund (2006) Core Questions on Drinking-Water and Sanitation for Household Surveys. Geneva: WHO and UNICEF.

30. ICF International (2011) Demographic and Health Survey Methodologies - Questionnaires: Household, Woman's, and Men's. MEASURE DHS Phase III. Calverton, MD: ICF International.

31. Luby SP, Halder AK, Huda TM, et al. (2011) Using child health outcomes to identify effective measures of handwashing. $A m \mathrm{~J}$ Trop Med Hyg 85, 882-892.

32. Pickering AJ, Davis J, Walters SP, et al. (2010) Hands, water, and health: fecal contamination in Tanzanian communities with improved, non-networked water supplies. Environ Sci Technol 44, 3267-3272.

33. United States Agency for International Development (2010) Access and Behavioral Outcome Indicators for Water, Sanitation, and Hygiene. Washington, DC: USAID.

34. Khatri R, Mishra S, Khanal V, et al. (2015) Factors associated with underweight among children of former-Kamaiyas in Nepal. Front Public Health 3, 11.

35. Centers for Disease Control and Prevention (2009) Preventing diarrheal disease in developing countries: safe storage of drinking water., http://www.ehproject.org/PDF/ehkm/safestorage2009.pdf (accessed November 2016).

36. Empowering the New Generation to Improve Nutrition and Economic opportunities (ENGINE) Team (2015) Nutritional and Health Related Outcomes of Children Treated for Severe Acute Malnutrition in a Community-Based Program. Jimma Zone: Valid International and Save The Children.

37. Pruss-Ustun A, Bartram J, Clasen T, et al. (2014) Burden of disease from inadequate water, sanitation and hygiene in lowand middle-income settings: a retrospective analysis of data from 145 countries. Trop Med Int Health 19, 894-905.

38. Strunz EC, Addiss DG, Stocks ME, et al. (2014) Water, sanitation, hygiene, and soil-transmitted helminth infection: a systematic review and meta-analysis. PLoS Med 11, e1001620.

39. Dangour AD, Watson L, Cumming O, et al. (2013) Interventions to improve water quality and supply, sanitation and hygiene practices, and their effects on the nutritional status of children. Cochrane Database Syst Rev, issue 8, CD009382.

40. Cichon B, Fabiansen C, Yaméogo CW, et al. (2016) Children with moderate acute malnutrition have inflammation not explained by maternal reports of illness and clinical symptoms: a cross-sectional study in Burkina Faso. BMC Nutr 2, 57.

41. Caulfield LE, de Onis M, Blössner M, et al. (2004) Undernutrition as an underlying cause of child deaths associated with diarrhea, pneumonia, malaria, and measles. Am J Clin Nutr 80, 193-198.

42. Dewey KG \& Mayers DR (2011) Early child growth: how do nutrition and infection interact? Matern Child Nutr 7, 129-142.

43. Petri WA Jr, Miller M, Binder $\mathrm{H}$, et al. (2008) Enteric infections, diarrhea, and their impact on function and development. J Clin Invest 118, 1277-1290.

44. O'Lorcain P \& Holland C (2000) The public health importance of Ascaris lumbriciodes. Parasitology 121, S51-S71. 